\title{
Pion Production at MINERvA
}

\author{
Alejandro Ramírez ${ }^{* \dagger}$ \\ Universidad de Guanajuato \\ E-mail: alejandro@fisica.ugto.mx
}

Pion production is one of the primary processes by which neutrinos are identified in running oscillation experiments such as Nova [1] and in future experiments like DUNE [2]. The MINERvA collaboration [3] has completed one NC (neutral current) and a few CC (charged current) measurements of this type using a $\mathrm{CH}$ (hydrocarbon plastic scintillator) target, from which four outstanding papers have been published. One of them is the $\mathrm{CC}$ coherent pion production, an important background for CCQE interactions in neutrino oscillation experiments, has been measured in both neutrino and anti-neutrino beams. The inelastic pion production cross section is much larger, and a more comprehensive data set for both $\pi^{+}$and $\pi^{0}$, also with neutrino and antineutrino beams is available. The other two are the $\mathrm{CC}$ single neutral pion and the NC diffractive neutral pion interactions, which are very rare and almost unstudied channels. All four reactions are also very interesting in their own right due to their potential to probe nuclear models.

Data compared with available theoretical calculations and models, are presented here.

To my parents Irma and José.

38th International Conference on High Energy Physics

3-10 August 2016

Chicago, USA

\footnotetext{
* Speaker.

On Behalf of the MINERvA Collaboration
} 


\section{Introduction}

The MINER $v$ A detector is upstream of the MINOS near detector [4] (used as a muon spectrometer) in the NuMI hall [5], at the Fermi National Accelerator Laboratory. It has already taken $v$ and $\bar{v}$ data, reaching $\sim 3.05 \times 10^{20}$ and $\sim 2.01 \times 10^{20}$ POT, respectively, with an average neutrino energy of $3.6 \mathrm{GeV}$. After upgrading the beamline, data is being taken at higher energies, $\left\langle\mathrm{E}_{v}\right\rangle=6.0 \mathrm{GeV}$, where new exciting results are to come.

MINER $v$ A's goal is to better understand the A-dependent dynamics of bound nucleons, in targets such as $\mathrm{He}, \mathrm{Fe}, \mathrm{C}$ and $\mathrm{Pb}$, helping in the testing of nuclear models. Therefore, understanding neutrino-induced pion interactions is crucial to constrain backgrounds and reduce systematics in those models, which in turn affect the uncertainties in neutrino oscillation experiments. One kind of such models, are the FSI (Final State Interactions) models, which account for the intra-nuclear re-scattering of the hadrons [6] [7].

\section{Charged Pion Production}

Most of the charged pions in the energy range considered here, are produced through resonances. The charged pion analysis is made of two sub-analyses: $v_{\mu}+C H \rightarrow \mu^{-}+1(N) \pi^{ \pm}+X$, selecting upper cuts in $W$ (hadronic invariant mass) of $1.4(1.8) \mathrm{GeV}$, separating single and multiple pion production, respectively. The 1-pion analysis is particularly helpful in testing theoretical calculations, due to the $\Delta(1232)$ dominance in this process. Results were compared with models and with the MiniBooNE results of the same measurement, which used a different flux and different target [6]. Pion angle and pion kinetic energy differential cross sections prefer models with FSI included (fig. 1). The agreement in magnitude with the MiniBooNE results for $\pi^{ \pm}$kinetic energies $>100 \mathrm{MeV}$ was otherwise unexpected, due to the differences in the $E_{v}$ and $W$ ranges in each measurement [6].
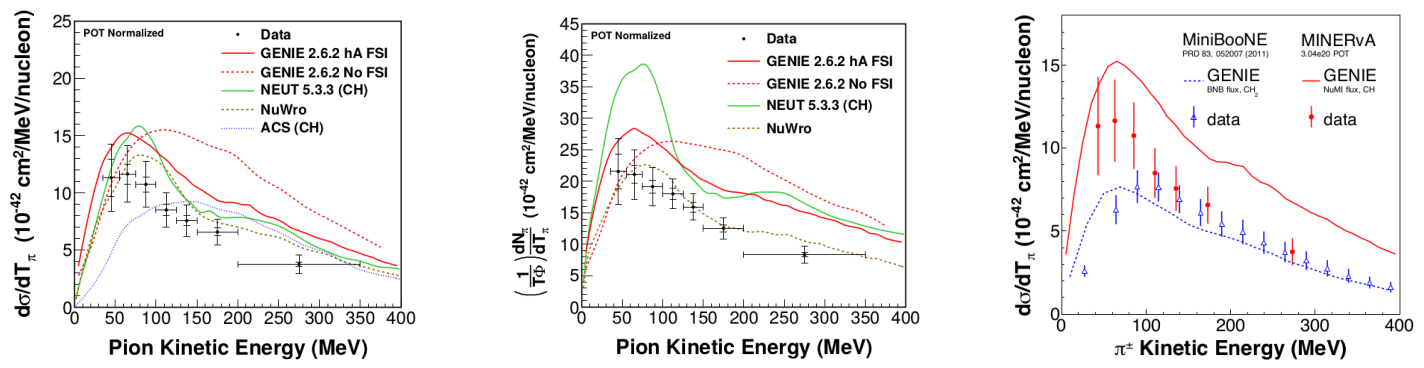

Figure 1: Charged pion results. a(b)) 1(N)Pion $T_{\pi}$ differential Xsec, c) MINERvA/MiniBooNE comparison.

\section{Coherent Pion}

By selecting events with only two tracks ( $\mu$ and $\pi^{ \pm}$), no evidence of nuclear breakup, and with low $|t|$ (square momentum transfer to the nucleus), neutrino and anti-neutrino cross sections of the coherent pion reaction: $v_{\mu}\left(\bar{v}_{\mu}\right)+C H \rightarrow \mu^{-(+)}+\pi^{+(-)}+C H$, were measured. There is a good agreement between cross sections, which suggests that the reaction is likely purely axial vector. 
The calculation of $|t|$, from directly measured kinematic variables of the muon and pion, allowed a model-independent measurement of the coherent differential cross section [8]. The model to which the data were compared to (Rein-Sehgal [8]), poorly reproduced the measured kinematics (fig. 2).
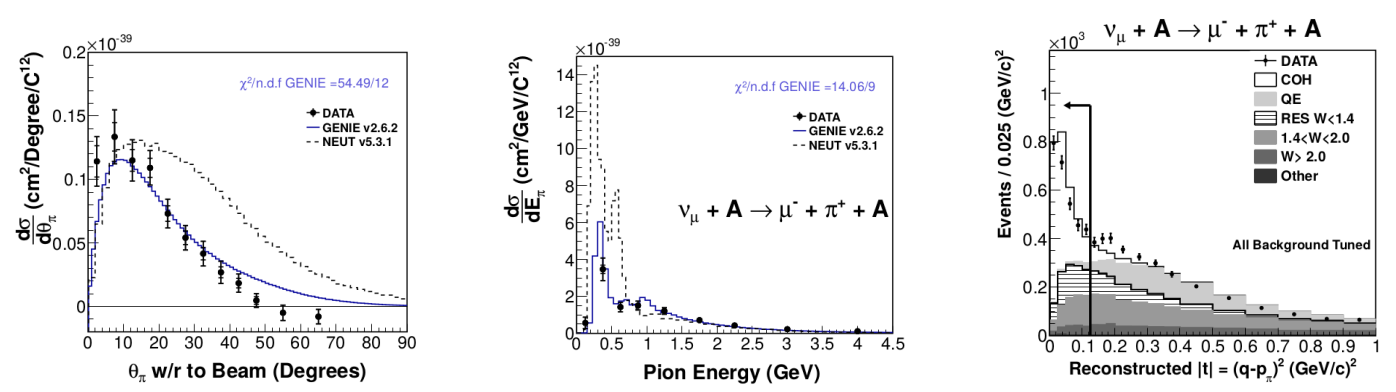

Figure 2: Coherent pion (neutrino) results. a) $\theta_{\pi}$ and $E_{\pi}$, b) differential Xsec, c) $|t|$ distribution.

\section{Single Neutral Pion}

This is the first measurement in detail of the kinematics of an almost unstudied reaction:

$\bar{v}_{\mu}+C H \rightarrow \mu^{+}+\pi^{0}+X$, where events with just one track (that of the positive muon) and with 2 photon candidates coming out of the main interaction vertex were selected. The $\pi^{0}$ can undergo different processes inside the nucleus: charge exchange, multi $\pi^{ \pm} \rightarrow \pi^{0}$, inelastic/elastic scattering, or can even not interact at all. Here is where the FSI models play an important role. All generators using FSI in the comparison, better reproduce the data (fig. 3). FSI effects are more pronounced in the $P_{\pi^{0}}$ than in the $\theta_{\pi^{0}}$, pointing out the contribution from the $\Delta(1232)$ resonance.
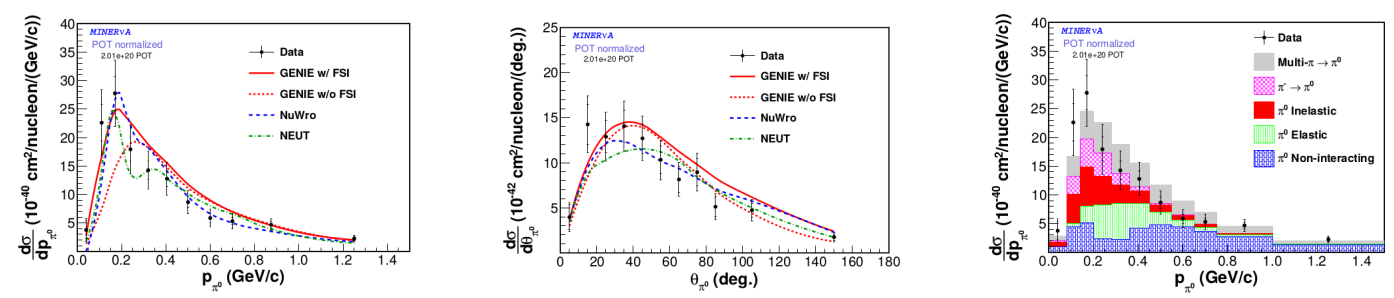

Figure 3: Neutral pion results. a) $p_{\pi^{0}}$ differential Xsec, b) $\theta_{\pi^{0}}$ differential Xsec, c) $T_{\pi}$ broken by type of interaction according to GENIE [6][8][7][9].

\section{Diffractive Neutral Pion}

In a previous measurement ( $v_{e}$ CCQE-like [9]) MINER $v$ A found an excess of EM showers, likely coming from $\gamma$ (photon) conversions. EM-like events were further studied using a $\mathrm{dE} / \mathrm{dx}$ analysis to check if they were consistent with a single $e^{ \pm}$or with $2 \gamma \mathrm{s}$. Sideband constraints reduce the possibility that the excess comes from: errors in the flux, background predictions or miss-modelling of the showers. Also single-particle studies suggest the showers are more likely due to $\gamma_{\mathrm{s}}$ from $\pi^{0}$ decays, and an extra upstream energy suggests the events come from diffractive scattering off $\mathrm{H}$ (fig. 4). Finally, the absence of $\mu^{ \pm} \mathrm{s}$ in the sample (muons exiting the back of the detector are rejected to avoid CC events) suggests that the reaction is $\mathrm{NC}\left(v_{e}+H \rightarrow v_{e}+\pi^{0}+H\right)$ [9]. 

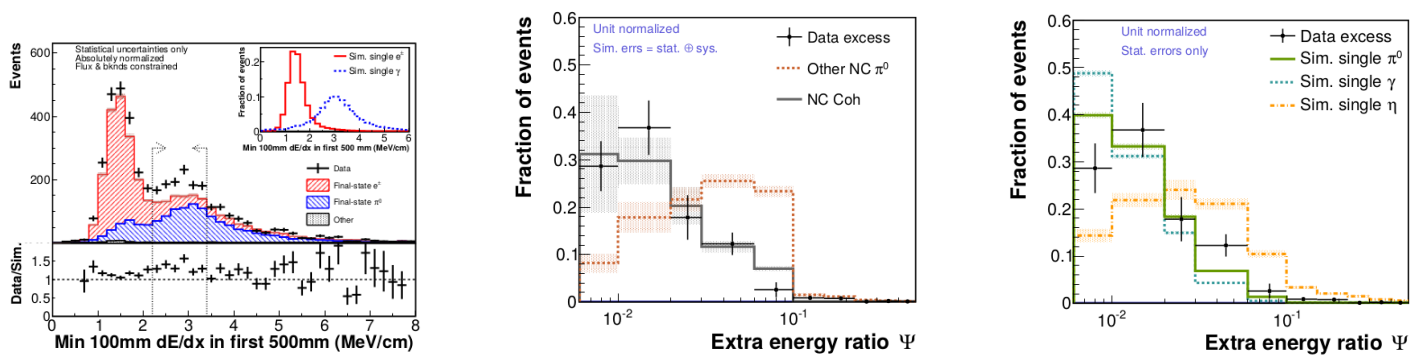

Figure 4: NC diffractive pion results. a) EM shower excess, b) Extra energy, NC and coherent - consistent, c) Extra energy, single $\pi^{0}$-consistent.

\section{Conclusions}

The MINERvA collaboration has released results on a variety of neutrino-induced pion reactions. Important not only for neutrino oscillations, but for model generators used in particle physics [6][7][8][9]. The rareness of 3 of these channels accounts for their low cross section and thereupon for the difficulty in "seeing" them. Nevertheless, due to the high statistics involved and the good resolution of its detector, MINERvA accomplished these measurements, helping to build better models.

\section{References}

[1] R.B. Patterson, The NOvA Experiment: Status and Outlook, Nucl.Phys.Proc.Suppl. 235-236 (2013) 151-157 [arXiv:1209.0716].

[2] R. Acciari, et al., Long-Baseline Neutrino Facility (LBNF) and Deep Underground Neutrino Experiment (DUNE): Volume 2: The Physics Program for DUNE at LBNF, FERMILAB-DESIGN (2015) 127 pp. (arXiv:1512.06148).

[3] L. Aliaga, et al., Design, Calibration, and Performance of the MINERvA detector, Nucl.Instrum.Meth. A743 (2014) 130-159 [arXiv: 1305 . 5199].

[4] C. Smith, et al., The MINOS Experiment, Nucl.Phys.Proc.Suppl. 143 (2005) 569-569

[5] P. Adamson et al., The NuMI Neutrino Beam, Nucl.Instrum.Meth. A806 (2016) 279-306 [arXiv:1507.06690].

[6] B. Everly, et al., Charged pion production in $v_{\mu}$ interactions on hydrocarbon at $\left\langle E_{v}\right\rangle=4.0 \mathrm{GeV}$, Phys.Rev.D 92 (2015) no. 9, 092008 [arXiv: 1406.6415$].$

[7] T. Le, et al., Single neutral pion production by charged-current $\bar{v}_{\mu}$ interactions on hydrocarbon at $\left.<E_{v}\right\rangle=3.6 \mathrm{GeV}$, Phys.Lett. B749 (2015) 130-136 [arXiv: 1503.02107 ].

[8] A. Higuera, et al., Measurement of Coherent Production of $\pi^{ \pm}$in Neutrino and Antineutrino Beams on Carbon from $E_{v}$ of 1.5 to $20 \mathrm{GeV}$, Phys.Rev.Lett. 113 (2014) no. 26, 261802 [arXiv: 1409.3835 ].

[9] J. Wolcott, et al., Evidence for neutral-current diffractive neutral pion production from hydrogen in neutrino interactions on hydrocarbon, Phys.Rev.Lett. 117 (2016) no. 11, 111801 [arXiv:1604.01728]. 